\title{
A deletion containing a CTCF-element in intron 8 of the $B b 57$ gene is partially responsible for juvenile obesity in the Berlin Fat Mouse
}

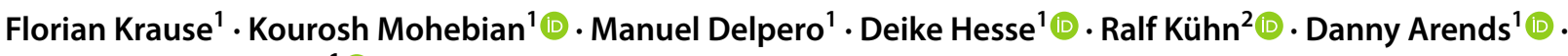 \\ Gudrun A. Brockmann ${ }^{1}$ (D)
}

Received: 5 August 2021 / Accepted: 29 November 2021 / Published online: 15 December 2021

(c) The Author(s) 2021

\begin{abstract}
The Berlin Fat Mouse Inbred (BFMI) line is a model for juvenile obesity. Previous studies on crosses between BFMI and $\mathrm{C} 57 \mathrm{Bl} / 6 \mathrm{~N}(\mathrm{~B} 6 \mathrm{~N})$ have identified a recessive defect causing juvenile obesity on chromosome 3 (jObes 1). Bbs 7 was identified as the most likely candidate gene for the observed effect. Comparative sequence analysis showed a $1578 \mathrm{bp}$ deletion in intron 8 of $B b s 7$ in BFMI mice. A CTCF-element is located inside this deletion. To investigate the functional effect of this deletion, it was introduced into B6N mice using CRISPR/Cas9. Two mice containing the target deletion were obtained (B6N Bbs $7^{\mathrm{emI} 8 \Delta 1}$ and $B b s 7^{\mathrm{emI} I 82}$ ) and were subsequently mated to BFMI and B6N to generate two families suitable for complementation. Inherited alleles were determined and body composition was measured by quantitative magnetic resonance. Evidence for a partial complementation (13.1-15.1\%) of the jObes 1 allele by the CRISPR/Cas9 modified B6N Bbs $7^{\mathrm{emI} 8 \Delta 1}$ and $B b s 7^{\mathrm{emI} I \Delta L 2}$ alleles was found. Mice carrying the complementation alleles had a 23-27\% higher fat-to-lean ratio compared to animals which have a B6N allele $\left(P_{(\mathrm{Bbs} 7 \mathrm{emI} 8 \Delta 1)}=4.25 \times 10^{-7} ; P_{(\mathrm{Bbs} 7 \mathrm{emI} 8 \Delta 2)}=3.17 \times 10^{-5}\right)$. Consistent with previous findings, the recessive effect of the BFMI allele was also seen for the B6N $B b s 7^{\mathrm{emI}} 8 \Delta 1$ and $B b s 7^{\mathrm{emI} I 82}$ alleles. However, the effect size of the B6N $B b s 7^{e m I 8 \Delta 1}$ and $B b s 7^{e m I 8 \Delta 2}$ alleles was smaller than the BFMI allele, and thus showed only a partial complementation. Findings suggest additional variants near $B b s 7$ in addition to or interacting with the deletion in intron 8 .
\end{abstract}

\section{Introduction}

The Berlin Fat Mouse Inbred line (BFMI) is a model for juvenile obesity. It was selected for high body fat content under standard diet (Wagener et al. 2006). BFMI mice have several features of the metabolic syndrome such as impaired lipid metabolism and reduced insulin sensitivity (Meyer et al. 2009; Hantschel et al. 2011; Heise et al. 2016). The BFMI mice develop obesity between 3 and 8 weeks (Wagener et al. 2006). Already under a standard breeding diet, BFMI males and females at 10 weeks have on average

Florian Krause and Kourosh Mohebian have contributed equally.

Gudrun A. Brockmann

gudrun.brockmann@agrar.hu-berlin.de

$1 \quad$ Albrecht Daniel Thaer-Institute for Agricultural and Horticultural Sciences, Humboldt-Universität zu Berlin, Unter den Linden 6, 10099 Berlin, Germany

2 Max-Delbrück-Center for Molecular Medicine in the Helmholtz Association, Robert-Rössle-Str. 10, 13125 Berlin, Germany
8.4 times more body fat mass than $\mathrm{C} 57 \mathrm{BL} / 6 \mathrm{NCrl}(\mathrm{B} 6 \mathrm{~N})$ reference mice (Wagener et al. 2006). A region on chromosome 3 (jObes 1 locus) was identified that explains $40 \%$ of the variation of fat mass in an $\mathrm{F} 2$ population from a cross between the obese BFMI and the lean B6N mouse line (Neuschl et al. 2010). Further fine mapping in an advanced intercross population generated from the original mapping population reduced the confidence interval to $\sim 370 \mathrm{~kb}$. Furthermore, complementation tests with different knockout mice in the target region revealed the Bardet Biedl syndrome 7 (Bbs7) gene as the most likely candidate for the juvenile obesity phenotype in BFMI mice (Arends et al. 2016).

Sequence comparisons between the BFMI and B6N mouse lines in the $B b s 7$ gene region showed a deletion of $1578 \mathrm{bp}$ in intron 8 of the $B b s 7$ gene $(3: 36,599,424-36,602,828)$ in BFMI mice (available from NCBI Sequence Read Archive (SRA) PRJNA717237). This deletion also leads to the loss of a binding site for the CCCTC-binding factor (CTCF). This CTCF-element in intron 8 was initially found by mapping DNase1 hypersensitive sites and by CTCF ChIP-Seq experiments in embryonic stem cells (Stadler et al. 2011). Such a CTCF-element may act as an insulator that is able to 
block the activity of enhancers through modification of the 3D DNA structure or act as a promoter activator or repressor (Phillips and Corces 2009). The binding of a CTCF-insulator binding protein was verified via the ChIP-Seq method (Chen et al 2008). ChIP-Seq can unveil a high range of binding sites for effectors such as CTCF-element, as it can recognize binding sites which exist at repetitive elements (Chen et al. 2008). Repetitive elements also occur around the CTCFelement in the deleted $B b s 7$ intron region. Re-mapping of the experimental data of Chen et al. in embryonic stem cells to the current genome of the mouse showed that CTCF binds indeed in intron 8 to the region of the deletion in the BFMI line (Ferrai et al. 2017).

In this study, the targeted generation of the BFMI deletion in intron 8 of $B b s 7$ using CRISPR/Cas9 (Ran et al. 2013) combined with complementation tests were used to identify the genetic effect of the deleted intron 8 region of $B b s 7$ on the development of obesity.

\section{Materials and methods}

\section{Mouse populations}

The Berlin Fat Mouse Inbred line 860-12 (BFMI) was used as our model for juvenile obesity. The line C57BL/6NCrl (B6N, Charles River Laboratories, Sulzfeld, Germany) served as lean control. These two lines have been used before for mapping the juvenile obesity QTL on mouse chromosome 3 (Neuschl et al. 2010; Arends et al. 2016).

\section{CRISPR/Cas9}

Whole genome sequencing showed a deletion of $1578 \mathrm{bp}$ $(3: 36,599,424-36,602,828)$ in intron 8 of $B b s 7$ in BFMI mice. This deletion was generated on a $\mathrm{B} 6 \mathrm{~N}$ background using CRISPR/Cas9 nuclease and a pair of single ( $\mathrm{sg}$ ) guideRNAs in single-cell mouse embryos as described in detail in (Wefers et al. 2017) and (Brandl et al. 2015). Consistent with the CRISPR/Cas9 endonuclease effect differences with respect to the strand breakpoints resulted in deletions of different lengths.

Briefly, Cas9 mRNA was prepared in a single step by in vitro transcription from plasmid pCAG-Cas9-162A linearized with AsiSI, AscI, and XbaI, using the mMessage mMachine T7 Ultra kit (Life Technologies, Ambion, AM1345, Life Technologies, Carlsbad, USA) (omitting the polyadenylation step). Cas9-162A mRNA was isolated using the Oligotex mRNA mini kit (cat No. 70022, Qiagen, Hilden, Germany). To produce the templates for sgRNA in vitro transcription, sgBbs 7 were amplified by PCR from guide RNA containing plasmids. As target sites, we selected two 20 nucleotide sequences located upstream of the deleted DNA segment (sgBbs7-A1: CCACTGTAT AGGGCGACCAC, sgBbs7-A2: AGGAGTGCTTGCCAC TGTAT) and two sequences located downstream (sgBbs7B1: CTACATAAAGAAGAGAGGTT, sgBbs7-B2: TAG TCTGTAAATCTCTTCAG).

$1 \mu \mathrm{g}$ template DNA was used for in vitro transcription using the Megashortscript kit (Ambion, cat No. AM1354) followed by the MEGAclear kit (Ambion, cat No. AM1908) for RNA purification. RNAs and targeting vectors were diluted in microinjection buffer $(10 \mathrm{mM}$ Tris, $0.1 \mathrm{mM}$ EDTA, pH 7.2) to the indicated working concentrations, filtrated through a centrifugal filter (Ultrafree, PFTE, Millipore, cat. no. UFC30LG25) and stored in single-use aliquots at $-80{ }^{\circ} \mathrm{C}$. For microinjections, zygotes were obtained by mating of C57BL/6 N males with superovulated C57BL/6 N females (Charles River, Sulzbach, Germany). Injected zygotes were transferred into pseudopregnant NMRI female mice to obtain live pups.

Sanger sequencing was performed to identify the exact DNA-sequence breakpoints (Fig. 1). All positions mentioned in this paper are based on GRCm38.p6 (Ensembl release 102).

The modified mouse lines were named C57BL/6N$B b s 7^{\text {emI } 8 \Delta^{*}}$, where emI $8 \Delta^{*}$ refers to an endonucleasemediated deletion of a specific part of intron 8 . Two male mice with partial deletions in intron 8 of the $B b s 7$ gene, comparable to the BFMI allele, were generated. These mice were used as founders for two separate and independent pedigrees. In each pedigree C $57 \mathrm{BL} / 6 \mathrm{~N}-B b s 7^{\mathrm{emI}} 8 \Delta^{*}$ founder mice were initially crossed with BFMI and B6N mice. Subsequent generations were produced by mating of siblings. We named the families with the obtained deletions I8 $\Delta 1$ (995 bp, 3:36,599,695-36,600,690) and $\mathrm{I} 8 \Delta 2(1,272 \mathrm{bp} ; 3: 36,599,578-36,600,850)$. Both C57BL/6N-Bbs $7^{\text {emI } 8 \Delta^{*}}$ mice and BFMI (1,578 bp; $36,599,687-36,601,265)$ share the deletion in intron 8 between 36,599,695 and 36.600,850 (Fig. 1). Two independent families were used since complementation in one of the two might allow to fine map which part of the deletion might be causal. All deletions caused the loss of a CTCF-binding site located at 3:36,599,801-36,600,200. Both male and female animals were included in the analysis.

\section{Husbandry conditions}

All mice were maintained under conventional conditions and a 12:12 h light:dark cycle (lights on from 6 a.m. to 6 p.m.) at a temperature of $22 \pm 2{ }^{\circ} \mathrm{C}$. Animals had ad libitum access to food and water. The mice were fed with a rodent standard diet (V1534-000 ssniff R/M-H; Ssniff Spezialdiäten GmbH, Soest, Germany). 


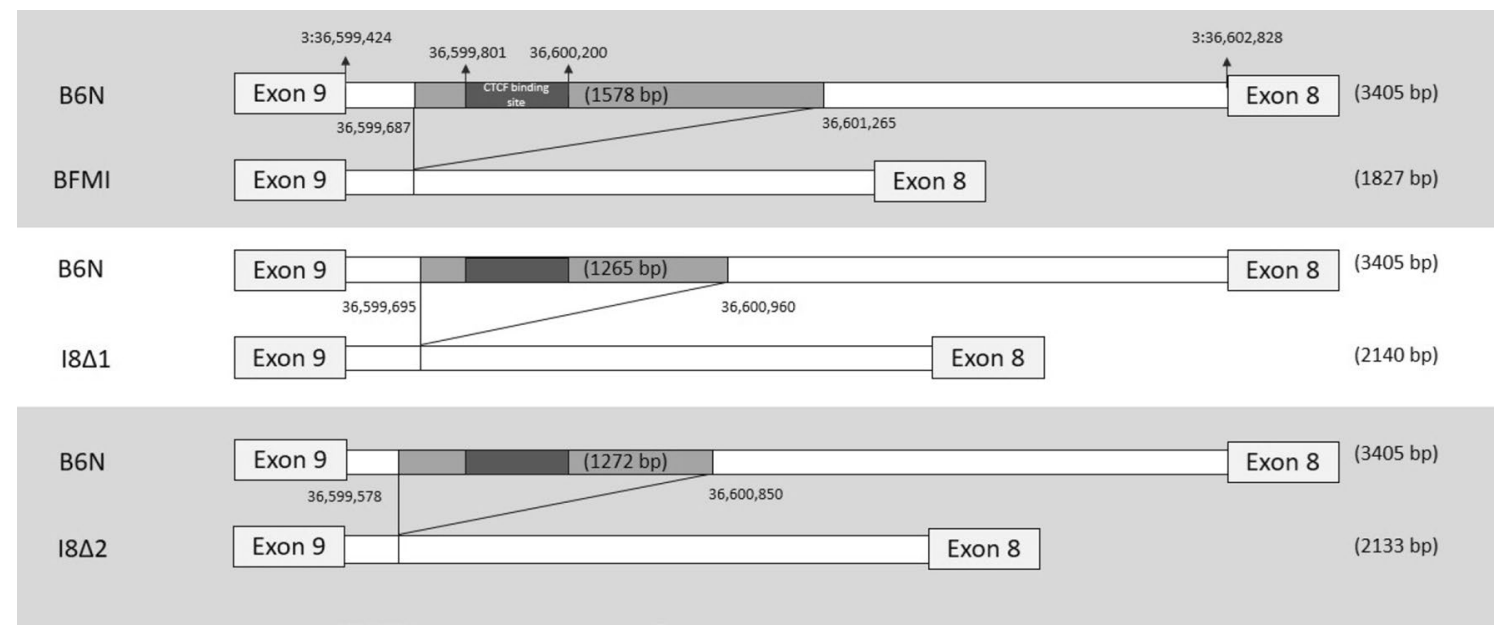

Fig. 1 Deletions in the intron 8 of Bbs7. Comparison of B6N (reference) to the naturally occurring deletion in the BFMI (top), and the two artificial CRISPR/Cas9 introduced deletions I8 $\Delta 1$ and I8 $\Delta 2$.

\section{Phenotyping}

Total fat mass and total lean mass were determined in nonanesthetized male and female mice at 10 weeks by quantitative magnetic resonance (QMR) using the EchoMRI whole body magnetic resonance analyzer (Echo Medical Systems, Houston, TX, USA) (Wagener et al. 2006). QMR measurements were performed in duplicates and the mean was used for further analyses. The ratio of total fat to total lean mass at 10 weeks (fat-to-lean ratio) was used to characterize the obese phenotype. Generations 7 to 14 of both independent families (I8 $\Delta 1$ and I8 $\Delta 2$ ) were analyzed in this paper for complementation, see supplemental Table 1 for an overview of numbers of animals (males/females) in each generation.

\section{Genotyping}

DNA was extracted from ear punches. Since three alleles occurred in our pedigrees, we generated a multiplex PCR system that allowed us to simultaneously genotype the BFMI and B6N alleles and the deletion alleles I $8 \Delta 1$ or I8 $\Delta 2$ (Supplementary Table 2). Using two forward and one reverse primer, we could distinguish all three alleles within each pedigree (BFMI, B6N, pedigree-specific CRISPR/Cas9 deleted alleles I $8 \Delta 1$ or I $8 \Delta 2$ ).

\section{Data analysis and complementation tests}

Phenotype and genotype data were analyzed using the $\mathrm{R}$ Project for Statistical Computing v4.0.2 (The R Development Core Team 2005). Phenotype measurements were adjusted for generation and sex since these external factors showed a significant effect on the phenotype. We performed
Please, note that the $B b s 7$ gene is on the negative strand, as such Exon 9 is in front of Exon 8

an ANOVA test with the general linear model (corrected for sex and generation) to identify significant differences of the fat-to-lean ratio between genotype classes. Subsequently, a Welch's $t$-test was used for pairwise group comparison. Differences between genotype classes were considered statistically significant at $P<0.05$.

\section{Results}

\section{Covariates (sex, generation, litter size)}

A linear model was used to investigate the influence of sex, generation, and litter size on the phenotype. The most significant effect was $\operatorname{sex}\left(P_{(\mathrm{I} 8 \Delta 1)}=2.20 \times 10^{-16}\right.$; $\left.P_{(\mathrm{I} 8 \Delta 2)}=5.95 \times 10^{-14}\right)$ followed by the generation effect $\left(P_{(\mathrm{I} 8 \Delta 1)}=0.066 ; P_{(\mathrm{I} 8 \Delta 2)}=0.002\right)$. Litter size showed no significant effect on the phenotype in both families $\left.{ }_{\left(P_{(\mathrm{I} 8 \Delta 1)}\right.}=0.158 ; P_{(\mathrm{I} 8 \Delta 2)}=0.453\right)$. As such, we corrected the fat-to-lean ratio for sex and generation. The adjusted fatto-lean ratio was used in further complementation testing.

\section{Family $1(18 \Delta 1)$}

The I8 $\Delta 1$ allele was tested for complementation with the BFMI allele. B6N allele carriers were used as lean reference. Homozygous B6N carriers were the smallest genotype group (8 animals; mean: $0.112 \pm 0.048$ ). However, there was no difference to the other two genotypes carrying one B6N allele $\left(P_{(\mathrm{B} 6 \mathrm{~N} / \mathrm{B} 6 \mathrm{~N} \text { vs. } \mathrm{BFMI} / \mathrm{B} 6 \mathrm{~N})}=0.879\right.$; $\left.P_{(\text {B6N/B6N vs. I8 } 1 / \mathrm{B} 6 \mathrm{~N})}=0.404\right)$. By taking this into account, combined with the knowledge that the effect is a recessive 
effect, these genotypes were merged $(\mathrm{x} / \mathrm{B} 6 \mathrm{~N})$ to create a larger reference panel consisting of 70 animals (mean: $0.096 \pm 0.035)$.

The two complementing genotypes BFMI/I $8 \Delta 1$ and $\mathrm{I} 8 \Delta 1 / \mathrm{I} 8 \Delta 1$ also showed no significant difference $\left(P_{(18 \Delta 1 / 18 \Delta 1 \text { vs. } B F M I / 18 \Delta 1)}=0.971\right)$ in fat-to-lean ratio and were also merged into a single complementation group $(y / I 8 \Delta 1)$ consisting of 440 animals (mean: $0.122 \pm 0.047)$. The positive control group (BFMI/BFMI) of family 1 (I8 $\Delta 1)$ contains 31 animals.

Complementation was investigated by comparing the $\mathrm{y} / \mathrm{I} 8 \Delta 1$ group to the positive control (BFMI/BFMI) and the negative control $(\mathrm{x} / \mathrm{B} 6 \mathrm{~N})$. This comparison showed a significant $\left(P_{(\mathrm{y} / 18 \Delta 1 \text { vs. } \mathrm{x} / \mathrm{B} 6 \mathrm{~N})}=4.25 \times 10^{-7}\right)$ increase in fat-to-lean ratio from 0.096 for $\mathrm{x} / \mathrm{B} 6 \mathrm{~N}$ animals to 0.122 for $y / I 8 \Delta 1$ animals. However, homozygous BFMI (mean: $0.268 \pm 0.102$ ) animals showed a significantly $\left(P_{(\mathrm{BFMI} / \mathrm{BFMI} \text { vs. y/I8 } 1)}=3.62 \times 10^{-9}\right)$ higher fat-to-lean ratio compared to the $y / I 8 \Delta 1$ group, pointing to a partial complementation (Fig. 2a, Supplementary Table 3).

The effects size, relative to the $\mathrm{x} / \mathrm{B} 6 \mathrm{~N}$ group, showed that the I $8 \Delta 1$ allele increases the fat-to-lean ratio by 0.026 , while the homozygous BFMI animals showed an increase of 0.172 . This indicates that the I8 $\Delta 1$ allele explains around $15.1 \%$ of the BFMI effect.

\section{Family 2 (18 182$)$}

Results from family 2 verify the results obtained in family 1 . However, it should be noted that the overall fat-to-lean ratio in family 2 is significantly lower than in family 1 (fat-to-lean (family2 vs. family1) $_{\text {) }}=-23.6 \%$, $P_{(\text {family2 vs. family 1) }}=5.68 \times 10^{-13}$ ).

Similar to family 1 , we also observed no difference between animals carrying one or more B6N alleles $\left(P_{(\mathrm{BGN} / \mathrm{B} 6 \mathrm{~N} \text { vs. BFMI/BGN })}=0.428 ; P_{(\mathrm{B} 6 \mathrm{~N} / \mathrm{B} 6 \mathrm{~N} \text { vs. } 18 \Delta 2 / \mathrm{B} 6 \mathrm{~N})}=0.953\right)$. Also, for the complementation group no difference was observed $\left(P_{(\mathrm{I} 8 \Delta 2 / 18 \Delta 2 \text { vs. BFMI/I8 } 82)}=0.146\right)$. Merging of groups led to a sample size of 68 animals in the $\mathrm{x} /$ B6N group and 310 animals in the $y / I 8 \Delta 2$ group. Furthermore, the same pattern as in family 1 was seen: $\mathrm{x} /$ B6N was the group with the lowest fat-to-lean ratio $(0.074 \pm 0.028), \mathrm{BFMI} / \mathrm{BFMI}$ showed the highest fat-tolean ratio $(0.204 \pm 0.112)$, and a significant increase of the $y / I 8 \Delta 2$ group $(0.091 \pm 0.032)$ compared to the $\mathrm{x} / \mathrm{B} 6 \mathrm{~N}$ group $\left(P_{(\mathrm{y} / 18 \Delta 2 \text { vs. } \mathrm{x} / \mathrm{B} 6 \mathrm{~N})}=3.17 \times 10^{-5}\right)$ was observed (Supplementary Table 4$)$. The I $8 \Delta 2$ allele showed a $13.1 \%$ partial complementation of the BFMI allele, an effect very similar to the I $8 \Delta 1$ allele.

The uncorrected data are also shown in the supplemental files (Supplemental Figs. 1 and 2).
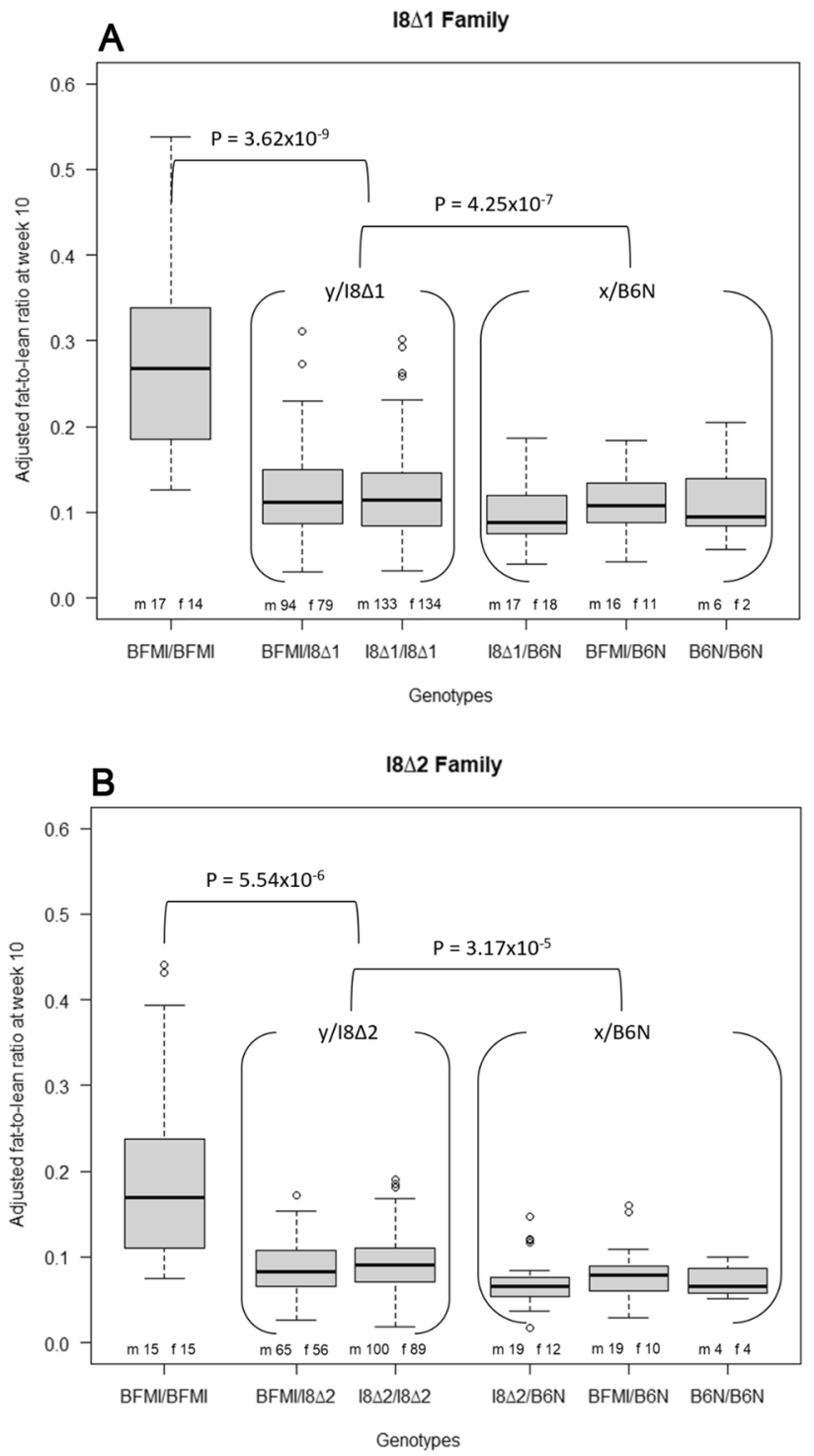

Fig. 2 a 541 animals of generations $7-14$ were analyzed in pedigree I $8 \Delta 1$. The gender breakdown is given on top of the $x$-axis for every genotype ( $m$ males; $f$ females). The phenotype on the $y$-axis was corrected for sex and generation. The homozygous BFMI genotype showed the highest fat-to-lean ratio of $0.268 \pm 0.102$. The $y / I 8 \Delta 1$ (grouping of I $\Delta \Delta 1 / \mathrm{I} 8 \Delta 1$ and BFMI/I8 $\Delta 2$ ) showed a fat-to-lean ratio of $0.122 \pm 0.047$, which was significantly lower $\left(P_{(\mathrm{BFMI} / \mathrm{BFMI} \text { vs. } \mathrm{y} / \mathrm{I} \Delta \Delta 1)}=3.62 \times 10^{-9}\right)$ than in BFMI homozygous mice, but also significantly higher significant $\left(P_{(\mathrm{y} / 18 \Delta 1 \text { vs. } \mathrm{x} / \mathrm{B} 6 \mathrm{~N})}=4.25 \times 10^{-7}\right)$ than genotypes of the reference group $\mathrm{x} / \mathrm{B} 6 \mathrm{~N}(0.096 \pm 0.035)(\mathrm{x} /$ B6N is the grouping of B6N/B6N, BFMI/B6N, and I8 $1 / \mathrm{B} 6 \mathrm{~N}$ ). The effect of the I8 $\Delta 1$ allele is $15.1 \%$ of the BFMI allele. b 408 mice of generation $7-14$ were analyzed in pedigree I8 $\Delta 2$. The phenotype on the $y$-axis was corrected for sex and generation. The homozygous BFMI genotype showed the highest fat-to-lean ratio of $0.204 \pm 0.112$. The $y / I 8 \Delta 2$ complementation group showed a fat-to-lean ratio of $0.091 \pm 0.032$, which was significantly lower $\left(P_{(\mathrm{BFMI} / \mathrm{BFMI} \text { vs. } \mathrm{y} / \mathrm{I8 \Delta 2})}=5.54 \times 10^{-6}\right)$ than in the BFMI homozygous mice, but significantly higher $\left(P_{(\mathrm{y} / 18 \Delta 2 \text { vs. } \mathrm{x} / \mathrm{B} 6 \mathrm{~N})}=3.17 \times 10^{-5}\right)$ than genotypes containing the $\mathrm{B} 6 \mathrm{~N}$ allele $(\mathrm{x} / \mathrm{B} 6 \mathrm{~N})$ with a fat-to-lean ratio of $0.074 \pm 0.028$. The effect of the I $8 \Delta 2$ allele is $13.1 \%$ of the BFMI allele 


\section{Discussion}

Both of the CRISPR/Cas9 modified mice and the BFMI mouse share the loss of a region in intron 8 of $B b s 7$ between $36,599,695$ and 3,600,960 base pairs on chromosome 3 containing a CTCF-element. The deletion of a CTCF-element is known to affect the 3D chromosomal structure (Phillips and Corces 2009) and as a result changes the accessibility of transcription factor binding sites (Ohlsson et al. 2001). Previously, it was observed that the Bbs7 expression in BFMI mice is significantly reduced compared to the expression of $B b s 7$ in $\mathrm{B} 6 \mathrm{~N}$ mice (Arends et al. 2016). Previous experiments on $B b s 7$ knockout mice showed obesity as one of the phenotypes observed in these mice (Zhang et al. 2013). The deleted CTCF-element in intron 8 in BFMI, I $8 \Delta 1$, and I $8 \Delta 2$ may be responsible for reducing $B b s 7$ transcription levels, which might cause obesity by influencing fat deposition.

Since we observed only a partial complementation in the I $8 \Delta 1$ and I8 22 families, one (or more) other variants need to be involved in causing the juvenile obesity phenotype of BFMI mice. Sequence data provided evidence for additional sequence variation between BFMI and B6N inside the jObes 1 locus. Eventually, one of the other sequence variants in BFMI in addition to the deletion in intron 8 is required for full complementation of the BFMI allele effect. These additional sequence variants did not occur in the CRISPR/Cas9 modified B6N mice. The additional sequence variants in BFMI are likely in close proximity to $B b s 7$, since otherwise recombinations would have caused BFMI variants in the background of the modified $\mathrm{B} 6 \mathrm{~N}$ mice in some animals of the $\mathrm{I} 8 \Delta 1$, and I $8 \Delta 2$ families. This would have led to a full complementation for some of the mice, which was not observed in our study. The additional sequence variants were not tested in this study. Therefore, we cannot exclude variants outside of the jObes 1 locus that could affect or modulate the juvenile obesity phenotype in BFMI mice directly or in interaction with the deleted region in intron 8 of $B b s 7$.

Several other mouse lines harbor a similar deletion found in BFMI mice. Some of these were previously investigated for complementation (NZO and AKR/J) (Arends et al. 2016). No complementation with NZO and AKR/J was detected in the 2016 study, and there it was concluded that the intron 8 deletion was not causal for the juvenile obesity. With the current finding of a partial complementation by I $8 \Delta 1$ and I8 $\Delta 2$, we revisit the intron 8 effect, and now show that part of the juvenile obesity seen in BFMI mice can be explained by the deletion in intron 8 . The previous study missed this partial complementation effect since the sample sizes used were only suitable for investigating full complementation.

\section{Conclusion}

We show that various partial deletions (I8 81 and I $8 \Delta 2$ ) in intron 8 of $B b s 7$ are able to partially complement the BFMI allele at the jObes 1 locus, seen by an increase in the fatto-lean ratio. Both I $8 \Delta 1$ and I8 $\Delta 2$ alleles show a recessive effect on the fat-to-lean ratio similar to the BFMI allele. The strengths of complementation of the I $8 \Delta 1$ and I $8 \Delta 2$ alleles are similar. By aligning the three deletions (I8 $\Delta 1$, I $8 \Delta 2$, and BFMI) we found the only common feature shared between the three deletions was a CTCF-element. Additionally, we conclude that another sequence variant at the jObes 1 locus in BFMI is essential to show the full effect on juvenile obesity as seen in BFMI mice. This is because (A) we see only partial complementation meaning another variant should be present, and (B) we know this variant needs to be close to the deletion otherwise recombinations in the two pedigrees would have caused around 50\% of offspring with the complementation genotypes to show full complementation due to inheritance of the second factor in the genetic background.

Supplementary Information The online version contains supplementary material available at https://doi.org/10.1007/s00335-021-09938-5.

Acknowledgements FK and KM were supported by the Deutsche Forschungsgemeinschaft (DFG) (BR 1285/12). We thank Angelika Ackermann for her assistance in the lab, Ben Gerhardt, Maximilian Sprechert, and Erik Leipe for genotyping. Marion Bütow, Michael Modrow, and Jens Fuchs for taking care of the animals and measuring phenotypes.

Author contributions FK and KM contributed equally, and both should be considered first authors. FK performed the statistical analysis, QMR measurements, and drafted the manuscript and figures. KM conducted most of the experiments, drafted the manuscript and figures. MD performed QMR measurements and provided feedback on the manuscript. DH critical reading of the manuscript and feedback. RK was responsible for CRISPR/Cas9 experiments and feedback on the manuscript. DA performed the statistical analysis, critical reading of the manuscript, and feedback. GB designed the experiment, critical reading of the manuscript, and feedback.

Funding Open Access funding enabled and organized by Projekt DEAL.

Data and code availability Scripts and raw data are available at https:// github.com/FlorianKrause/Bbs7-complementation.

\section{Declarations}

Conflict of interest The authors declare no conflict of interest.

Ethical approval All experimental treatments of mice were approved by the German Animal Welfare Authorities (Approval No. G0162/12, G0292/15, G0302/15 and G0174/19).

Open Access This article is licensed under a Creative Commons Attribution 4.0 International License, which permits use, sharing, 
adaptation, distribution and reproduction in any medium or format, as long as you give appropriate credit to the original author(s) and the source, provide a link to the Creative Commons licence, and indicate if changes were made. The images or other third party material in this article are included in the article's Creative Commons licence, unless indicated otherwise in a credit line to the material. If material is not included in the article's Creative Commons licence and your intended use is not permitted by statutory regulation or exceeds the permitted use, you will need to obtain permission directly from the copyright holder. To view a copy of this licence, visit http://creativecommons. org/licenses/by/4.0/.

\section{References}

Arends D, Heise S, Kärst S et al (2016) Fine mapping a major obesity locus (jObes1) using a Berlin Fat Mouse $\times$ B6N advanced intercross population. Int J Obes 40:1784-1788. https://doi.org/ 10.1038/ijo.2016.150

Brandl C, Ortiz O, Röttig B et al (2015) Creation of targeted genomic deletions using TALEN or CRISPR/Cas nuclease pairs in onecell mouse embryos. FEBS Open Bio 5:26-35. https://doi.org/ 10.1016/j.fob.2014.11.009

Chen X, Xu H, Yuan P et al (2008) Integration of external signaling pathways with the core transcriptional network in embryonic stem cells. Cell 133:1106-1117. https://doi.org/10.1016/j.cell.2008.04. 043

Ferrai C, Torlai Triglia E, Risner-Janiczek JR et al (2017) RNA polymerase II primes polycomb-repressed developmental genes throughout terminal neuronal differentiation. Mol Syst Biol 13:946. https://doi.org/10.15252/msb.20177754

Hantschel C, Wagener A, Neuschl C et al (2011) Features of the metabolic syndrome in the Berlin Fat Mouse as a model for human obesity. Obes Facts 4:270-277. https://doi.org/10.1159/00033 0819

Heise S, Trost J, Arends D et al (2016) High variability of insulin sensitivity in closely related obese mouse inbred strains. Exp Clin Endocrinol Diabetes 124:519-528. https://doi.org/10. 1055/s-0042-109261
Meyer CW, Wagener A, Rink N et al (2009) High energy digestion efficiency and altered lipid metabolism contribute to obesity in BFMI mice. Obesity 17:1988-1993. https://doi.org/10.1038/oby. 2009.124

Neuschl C, Hantschel C, Wagener A et al (2010) A unique genetic defect on chromosome 3 is responsible for juvenile obesity in the Berlin Fat Mouse. Int J Obes 34:1706-1714. https://doi.org/10. 1038/ijo.2010.97

Ohlsson R, Renkawitz R, Lobanenkov V (2001) CTCF is a uniquely versatile transcription regulator linked to epigenetics and disease. Trends Genet 17:520-527

Phillips JE, Corces VG (2009) CTCF: master weaver of the genome. Cell 137:1194-1211

Ran FA, Hsu PD, Wright J et al (2013) Genome engineering using the CRISPR-Cas9 system. Nat Protoc 8:2281-2308. https://doi.org/ 10.1038/nprot.2013.143

Stadler MB, Murr R, Burger L et al (2011) DNA-binding factors shape the mouse methylome at distal regulatory regions. Nature 480:490-495. https://doi.org/10.1038/nature10716

The R Development Core Team (2005) R: A language and environment for statistical computing. In: $\mathrm{R}$ foundation for Statistical Computing

Wagener A, Schmitt AO, Aksu S et al (2006) Genetic, sex, and diet effects on body weight and obesity in the Berlin Fat Mouse Inbred lines. Physiol Genom 27:264-270. https://doi.org/10.1152/physi olgenomics.00225.2005

Wefers B, Bashir S, Rossius J et al (2017) Gene editing in mouse zygotes using the CRISPR/Cas9 system. Methods 121-122:5567. https://doi.org/10.1016/j.ymeth.2017.02.008

Zhang Q, Nishimura D, Vogel T et al (2013) BBS7 is required for BBSome formation and its absence in mice results in Bardet-Biedl syndrome phenotypes and selective abnormalities in membrane protein trafficking. J Cell Sci 126:2372-2380. https://doi.org/10. $1242 /$ jcs. 111740

Publisher's Note Springer Nature remains neutral with regard to jurisdictional claims in published maps and institutional affiliations. 